\title{
BILATERAL DOUBLE BELLIES OF ABDUCTOR POLLICIS LONGUS
}

\section{Gargi Soni *1, B Ravindra Kumar 2a,2b.}

${ }^{*_{1}}$ Head, Department of Anatomy, International Medical School, Management \& Science University, Malaysia.

2a, Former Lecturer, Department of Anatomy, International Medical School, Management \& Science University, Malaysia.

${ }^{2 b}$ Research Scholar, Department of Anatomy, Sri Padmavathi Medical College for Women, SVIMS University, Tirupati, Andhra Pradesh, India.

\section{ABSTRACT}

Introduction: The variations in the insertion and number of tendons of abductor pollicis longus are very common and have been highlighted by many researchers, but the variations in the origin of abductor pollicis longus (APL) have not received much attention.

Materials: During the routine dissection of 12 upper limbs from six cadavers in the Management and Science University, Malaysia, bilateral double bellies at origin of abductor pollicis longus were found in ten limbs and in all twelve limbs a double tendon of abductor pollicis longus was found. The additional muscle bellies in all the cases arose from the lateral aspect of the distal portion of abductor pollicis longus muscle. The lateral tendon, lying adjacent to the extensor pollicis brevis tendon, was considered the main apl tendon, while the medial tendons were regarded as accessory ones.

Observations: The lateral tendons were inserted into the base of the first metacarpal bone; to its anterolateral surface in 8 limbs and on its lateral surface in 4 limbs. The medial accessory tendons of the APL had different sites of insertions at: the anterolateral surface of base of 1st metacarpal bone in 8 limbs and on trapezium in four limbs.

Conclusion: The knowledge of presence of accessory tendons of APL is important for the hand surgeon not only to prevent unintentional injuries but also to be able to use these accessory tendons as grafting material and also the accessory tendons of APL contribute to the development of de quervain's tenosynovitis.

KEY WORDS: Abductor Pollicis, Double tendons, Double Bellies.

Address for Correspondence: Dr. Gargi Soni, Head, Department of Anatomy, International Medical School, Management \& Science University, Malaysia. E-Mail: bhasingargi@rediffmail.com

\section{Access this Article online}

\section{Quick Response code}

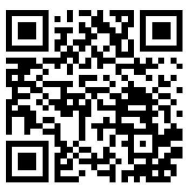

DOI: $10.16965 /$ ijar.2017.397

Web site: International Journal of Anatomy and Research

ISSN 2321-4287

www.ijmhr.org/ijar.htm

Received: 07 Aug 2017

Peer Review: 07 Aug 2017

Revised: None
Accepted: 03 Oct 2017

Published (O): 01 Nov 2017

Published (P): 01 Nov 2017

\section{INTRODUCTION}

The presence of additional muscle bellies and tendons are of importance to the surgeon as these variations may lead to errors during surgery and also the awareness regarding such variations may be of help to the hand surgeon as these accessory structures might serve as potential graft material.

The variations in the number and site of insertion of the APL tendon have been studied by many previous researchers; 2-9 tendons of APL have been reported with the insertions at the base of 1st metacarpal, trapezium and attached to thenar muscles [1-7]. 
The presence of accessory tendons is also an important contributing factor in the development of de quervain's tenosynovitis [4].

However, the literature regarding the variations in the origin of apl is scanty and only a few case reports and studies regarding the double bellies of abductor pollicis longus could be found.

The knowledge of presence of accessory muscle bellies and tendons of APL is important for the hand surgeon not only to prevent unintentional injuries but also to be able to use these accessory tendons as grafting material.

\section{MATERIALS AND METHODS}

During the routine dissection of twelve upper limbs from six cadavers in the Management and Science University, Malaysia, bilateral double bellies of abductor pollicis longus were found in ten limbs and in all twelve limbs a double tendon of abductor pollicis longus was found.

\section{OBSERVATIONS}

The additional muscle bellies in all the cases arose from the lateral aspect of the distal portion of abductor pollicis longus muscle. The lateral, lying adjacent to the extensor pollicis brevis tendon, was considered the main APL tendon, while the medial tendons were regarded as accessory ones, according to classification given by mehta et al [8]. The lateral tendons were inserted into the base of the first metacarpal bone; to its anterolateral surface in 8 limbs and on its lateral surface in 4 limbs. The medial accessory tendons of the apl had different sites of insertions at: the anterolateral surface of base of 1st metacarpal bone in 6 limbs, on trapezium in 5 limbs and was attached to thenar muscles (abductor pollicis brevis, opponens pollicis and flexor pollicis brevis) in one limb.

Fig. 1, 2, 3 and 4: Showing the double bellies and tendon of the Abductor Pollicis Longus Muscle (APL).
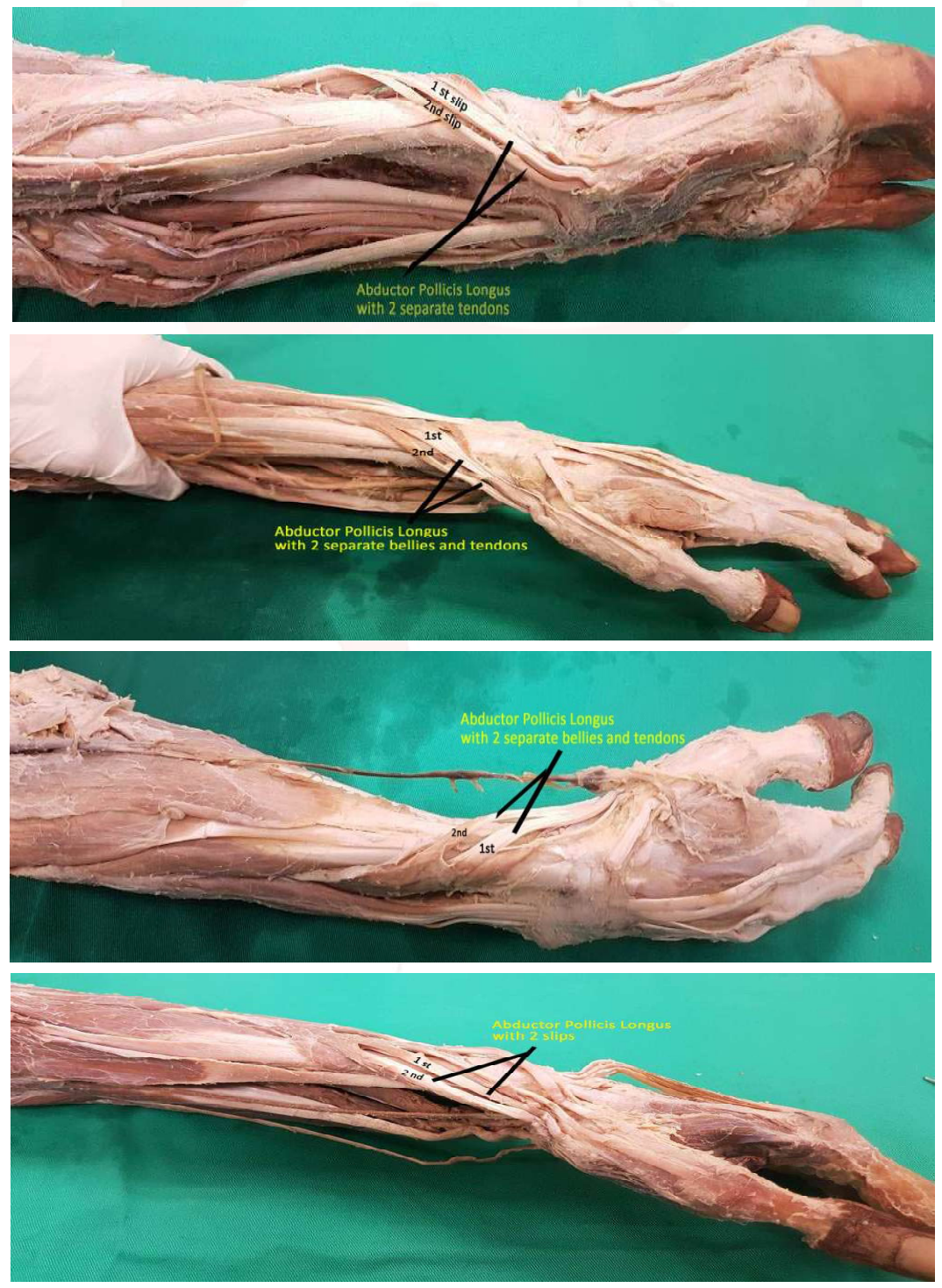


\section{DISCUSSION}

In the present study we found that the accessory abductor pollicis longus tendons had their own muscle belly that arose from the lateral aspect of the distal portion of abductor pollicis longus muscle in ten limbs amongst the twelve dissected limbs.

Fabrizio and clemente found the presence of an additional muscular venter from the lateral and distal portion of the abductor pollicis longus and inserting in a variable way. Fabrizio and clemente found this variation on the forearm of 15 cadavers in a total of 50 dissected cadavers [20\%] and according to them it can be a factor of contribution to the etiology of the syndrome of intersection [9]. The intersection syndrome is due to overuse tenosynovitis that occurs around the intersection of the first extensorm compartment (abductor pollicis longus and extensor pollicis brevis) and second extensor compartment (extensor carpi radialis longus and extensor carpi radialis brevis) within the forearm.

Bravo et al based on their dissections in 78 limbs, reported double muscle bellies of apl in $16 \mathrm{limbs}$ that is in $20 \%$ of the cases [10]. Rao et al in their study found one case of double muscle belly of abductor pollicis longus [11].

In the present case the presence of double bellies of abductor pollicis longus in ten of the twelve limbs dissected shows a much higher incidence of the presence of double muscle bellies of abductor pollicis longus muscle as compared to the previous studies which have reported its incidence to be $20 \%$, and this needs the attention of hand surgeons and orthopedicians.

The APL muscles is the stabilizer of the carpometacarpal joint and the presence of bi-tendinious, double muscle bellies might be a factor contributing to mechanical strength of the muscle. The apl tendon can be used in various surgeries such as a tendon transfer in cases of osteoarthritis of the base of the thumb or in extensor tendon injuries. The accessory muscle bellies are commonly asymptomatic and usually an incidental finding. However, in some cases accessory muscles may produce clinical symptoms related to palpable swelling, or may be the result of mass effect on neurovascular structures, typically in fibro-osseous tunnels [12]. Coleman et al reported that a single tendon of apl is odd in 175 examined specimens [4]. Beshbishy and hamid also reported the absence of single tendons of apl in the fifty limbs which they studied. They noted the apl to have 2-6 tendons of which the double tendon was found to be the most common i.e. In $40 \%$ of the cases [13]. Roy et al and tewari et al in their respective studies from the indian subcontinent also reported the bi-tendinous slips of apl as the most common pattern of apl arrangement on dorsum of the wrist $[14,15]$. Thwin et al found supernumerary tendon slips of the apl, ranging from 3 to 14 slips, in the ten upper limb specimens dissected, in a study conducted in malaysia [16].

In the present study also two tendons of abductor pollicis longus were found in all twelve limbs. The medial accessory tendons of the apl had different sites of insertions at: the anterolateral surface of base of 1st metacarpal bone in 6 limbs, on trapezium in 5 limbs and was attached to thenar muscles (abductor pollicis brevis, opponens pollicis and flexor pollicis brevis) in one limb. These studies including the present study suggest that double tendon of abductor pollicis longus muscle seems to be more common than a single tendon, so should we consider the double tendon a variation or a normal pattern? The presence of double muscle bellies of abductor pollicis longus with double tendon of apl seems to be common in the dorsal compartment of the wrist in malaysian population, however, the literature regarding the prelevance of this variation in Malaysian population is scanty and further studies in larger sample size are required to know its incidence. Thus, it is important for the malaysian orthopedic and hand surgeons to understand this variation as it would be helpful in reconstructive surgeries of hand and also in the diagnosis and treatment of de quervain's disease and intersection syndrome.

\section{Conflicts of Interests: None}

\section{REFERENCES}

[1]. Lacey T, 2nd, Goldstein La, Tobin Ce. Anatomical and clinical study of the variations in the insertions of the abductor pollices longus tendon, associated with stenosing tendovaginitis. J bone joint surg am. 1951; 33-a: 347-50. 
[2]. Rousset P, Vuillemin-Bodaghi V, Laredo Jd, ParlierCuau C. Anatomic variations in the first extensor compartment of the wrist: accuracy of us. Radiology. 2010; 257:427-33.

[3]. Choi Sj, Ahn Jh, Lee Yj, Ryu Ds, Lee Jh, Jung Sm, Et Al. De quervain disease: us identification of anatomic variations in the first extensor compartment with an emphasis on subcompartmentalization. Radiology. 2011; 260:480-6.

[4]. Coleman Ss, Mcafee Dk, Anson Bj. The insertion of the abductor pollicis longus muscle; an anatomical study of 175 specimens. Q bull northwest univ med sch. 1953; 27:117-22.

[5]. Dhuria R, Mehta V, Suri Rk, Rath G. Anomalous composition of musculature of the first dorsal fibroosseous compartment of the wrist. Singapore med j. 2012;53:e133-5.

[6]. Nayak Sr, Krishnamurthy A, Pai Mm, et al. Multiple variations of the extensor tendons of the forearm. Rom j of morpho and embryol. 2008;49:97100.

[7]. Akan M, Gideroglu K, Cakir B. Multiple tendons of the abductor pollicis longus muscle. Hand surg. 2002; 7:289-91.

[8]. Mehta V, Arora J, Suri Rk, Rath G. A rare quadruplicate arrangement of abductor pollicis longus tendons: anatomical clinical orthopaedics and related research.and clinical relevance. Clinics (sao paulo) 2009;64:153-5.

[9]. Fabrizio Pa, Clemente Fr. A variation in the organization of abductor pollicis longus. Clin anat. 1996; 9:371-5.
[10]. Bravo E, Barco R, Bullón A. Anatomic study of the abductor pollicis longus: a source for grafting material of the hand. 2010;468(5):1305-1309. Doi:10.1007/s11999-009-1059-4.

[11]. Rao Mk, Vollala Vr, Bhat Sm, Bolla S, Samuel Vp, Pamidi N. Four cases of variations in the forearm extensor musculature in a study of hundred limbs and review of literature. Indian j plast surg 2006;39:141-7.

[12]. Sookur Pa, Naraghi Am, Bleakney Rr, Jalan R, Chan $\mathrm{O}$, White Lm. Accessory muscles: anatomy, symptoms, and radiologic evaluation. Radiographics, 2008;28:481-499.

[13]. El-Beshbishy Ra, Abdel-Hamid Ga. Variations of the abductor pollicis longus tendon: an anatomic study. Folia morphol (warsz) .2013; 72:161-6.

[14]. Roy Aj, Roy An, De C, Banerji D, Das S, Chatterjee B, et al. A cadaveric study of the first dorsal compartment of the wrist and its content tendons: anatomical variations in the indian population. $J$ hand microsurg. 2012; 4:55-9.

[15]. Tewari J, Mishra Pr, Tripathy Sk. Anatomical variation of abductor pollicis longus in indian population: a cadaveric study. Indian j orthop. 2015; 49:549-53.

[16]. Thwin SS, Zaini F, Than M. Multiple variations of the tendons of the anatomical snuffbox. Singapore medical journal. 2014;55(1):37-40. Doi:10.11622/ smedj.2013216.

How to cite this article:

Gargi Soni, B Ravindra Kumar. BILATERAL DOUBLE BELLIES OF

ABDUCTOR POLLICIS LONGUS. Int J Anat Res 2017;5(4.2):4566-

4569. DOI: $10.16965 /$ ijar.2017.397 